\title{
Autonomous Learning Multiple-Model Zero-Order Classifier for Heart Sound Classification
}

\author{
Eduardo Soares ${ }^{\mathrm{a}}$, Plamen Angelov ${ }^{\mathrm{a}}, \mathrm{Xiaowei} \mathrm{Gu}^{\mathrm{a}}$ \\ ${ }^{a}$ School of Computing and Communications, Lancaster University, Lancaster, LA1 4WA, \\ $U K$ \\ E-mail: e.almeidasoares@lancaster.ac.uk; p.angelov@lancaster.ac.uk; x.gu3@lancaster.ac.uk
}

\begin{abstract}
This paper proposes a new extended zero-order Autonomous Learning Multiple2 Model (ALMMo- $0^{*}$ ) neuro-fuzzy approach in order to classify different heart 3 disorders through sounds. ALMMo-0* is build upon the recently introduced 4 ALMMo-0. In this paper ALMMo-0 is extended by adding a pre-processing 5 structure which improves the the performance of the proposed method. ALMMo$60^{*}$ has as a learning engine composed of hierarchical a massively parallel set of 7 0-order fuzzy rules, which are able to self-adapt and provide transparent and 8 human understandable $I F$... THEN representation. The heart sound recordings considered in the analysis were sourced from several contributors around 10 the world. Data were collected from both clinical and nonclinical environment, 11 and from healthy and pathological patients. Differently from mainstream ma12 chine learning approaches, ALMMo- $0^{*}$ is able to learn from unseen data. The main goal of the proposed method is to provide highly accurate models with high transparency, interpretability, and explainability for heart disorder diagnosis. Experiments demonstrated that the proposed neuro-fuzzy-based modeling is an efficient framework for these challenging classification tasks surpassing its state-of-the-art competitors in terms of classification accuracy. Additionally, ALMMo-0* produced transparent AnYa type fuzzy rules, which are human interpretable, and may help specialists to provide more accurate diagnosis. Medical doctors can easily identify abnormal heart sounds by comparing a patient's sample with the identified prototypes from abnormal samples by ALMMo-0*.
\end{abstract}

Preprint submitted to Applied Soft Computing

June 4, 2020 
Keywords:

Autonomous Learning, Data Clouds, Evolving Fuzzy Systems, Heart Sound Classification, Rule-Based System.

\section{Introduction}

The development of models able to efficiently classify values is a hard and of great importance task in a variety of knowledge domains [1]. In the case of heart sound classification it is of vital importance as most of the heart valve disorders are reflected to heart sounds [2]. Heart sounds are characterized by low frequency signals produced by heart valves [3]. However, disorders caused by turbulence in the blood circulation through contracted heart valves or reflow through the valves between atria and ventricles cause high frequency sounds. Such abnormal sound is known as murmur [4].

According to [5], cardiovascular disease is one of the leading cause of morbidity and mortality worldwide with an estimated 17.9 million, or $31.0 \%$ of the global population, have died from cardiovascular diseases related conditions in 2017. In low to middle income countries, this situation is particularly alarming, as high quality diagnostics can be often difficult to obtain, due to its high costs in these regions [6]. As stated in [7], heart sounds may include indicators of disorder, or warnings about future disorders. These indicators may be present during at all time occurring throughout the whole signal, or can occur randomly. Accurate heart sound classification allows more time for emergency management, preparation and mobilization of resources for recovery, and may save many lives 8]. Additionally, better classification results improve the predictions of other metrics such as blood pressure [9].

As the quality of monitoring data has improved over the years [6], data become indispensable in operational heart sound classification models. However, uncertainty contained in numerical models vary substantially as heart sounds change their pattern due to complex and highly nonstationary nature of heart sound signals, with negative effects on the quality of the classification task [10]. 
Advanced classifiers have been proposed specifically for heart sound classification, such as neural-network-based [3, and support vector machine [11, 12] classifiers. However, if they are not equipped with evolving algorithms capable of adapting their parameters and structure, then they are not able to capture certain time-varying properties of nonstationary heart sound conditions and features of a rich variety of vibrations of the heart and blood flow [6]. Although, several studies have been conducted the digital recording of heart sounds, named as phonocardiogram (PGC). There is a lack of studies using transparent rulebased approaches that provide explainable and interpretable results for specialists [13, 14, 15. Most of the existing studies are with 'black box' approaches or very complex models [16, 17, 18.

Moreover, as the volume of the data collected continuously with a fast rate has increased due to the advent of the Internet of Things (IoT), automation of complex systems, and proliferation of small-scale computing devices, data stream processing has become an issue of primary importance 19, 20. A way to deal with such large volumes of data is through the use of a class of computational methods known as evolving intelligent systems [21, 22, 23, 24, 25, 26]. The evolving approach is an effective and efficient way of handling data streams due to its ability to adapt models to different situations and provide quick response to changes [27, 28]. Evolving systems have demonstrated great ability to deal with medical applications as one can see in 229, 30, 31, 32.

A granular neural network framework for evolving fuzzy system is introduced by 29 and it demonstrated great ability to deal with Parkinson's symptom prediction surpassing its competitors in terms of accuracy due to its ability to adapt itself on a non-stationary environment. According to 30 spiking neural networks have revealed themselves as one of the most successful approaches to model the behavior and learning potential of the brain, and exploit them to undertake practical online learning tasks due to its evolving ability. Moreover, 31 has shown that eClass can effectively be applied to the classification of diabetes and dermatological diseases from discrete numerical samples.

This paper we propose a new a new method to autonomously classify ab- 
normal heart disorders through sounds. It builds upon the recently introduced zero-order Autonomous Learning Multiple-Model (ALMMo-0) classifier [33, 34. The ALMMo-0 classifier is significantly extended as we add a standardization and normalization pre-processing structure. The pre-processing block helps to improve the accuracy of the classifier as it creates more stable models [35]. The proposed approach has a learning mechanism composed of a massively parallel set of 0-order fuzzy rules, which are able to self-adapt and provide transparent and human understandable IF ... THEN representation 36. It is also able to self-evolve its structure and self-update its meta-parameters as newly observed training images arrive from the data stream, which makes the classifier applicable for real-time applications 37, 38. Due to its evolving structure, the proposed method is able to deal with large volumes of data, avoiding the curse of dimensionality.

A 'PhysioNet' dataset was considered in the analysis. The 'Phisionet' dataset is composed of eight independent heart sound databases sourced from several contributors around the world. Data were collected from either clinical and nonclinical environment, and from healthy and pathological patients. Both healthy and pathological patients include children and adults 39. The dataset is provided by [39], and it was used in the 'Computing in Cardiology Challenge', which is the major challenge involving computing and cardiology.

In brief, the main contributions of this paper are:

- It offers a new method to automatically classify heart disorders through sounds.

- An extended version of the recently zero-order Autonomous Learning Multiple-Model (ALMMo-0) classifier with a improved pre-processing block.

- A human-interpretable, computationally efficient classifier outperforming the competitors.

The remainder of this paper is structured as follows. Section II presents the proposed extended Zero-order Autonomous Learning Multiple-Model (ALMMo- 
$\left.0^{*}\right)$ system classification approach. Section III describes the methodology employed in the analyses, and the performance indexes used for comparison. Results and discussions are shown in Section IV. Conclusion and future research directions are given in Section $\mathrm{V}$.

\section{ALMMo-0* Neuro-Fuzzy System}

Traditionally the pipeline of learning from data has the following steps:

1)Pre-precessing, which includes different substeps like normalization, standardization, dealing with missing data, and feature selection [40. Specifically for image processing there are often other stages, such as rotation, augmentation, scaling, elastic deformation, etc 41. Even deep learning methods which claims to avoid handcrafting applies some of the cited steps.

2)Learning phase, which can be offline, when the complete dataset is available; or it can be done online, when the data arrives in the form of data streams (sample-by-sample). Evolving learning, ability of the algorithms to adapt their parameters and structure according to data streams, is non sophisticated form of online learning [42, 28,

3)Generating outputs for new unseen data, which is the validation phase. Different algorithms use different strategies in order to validate the model generated in the learning phase.

The proposed method also starts with a pre-processing step which involves mostly the same steps depending on the specific problem, for example, for image processing we may also apply scaling, augmentation, rotation. Practically for all problems normalization and standardization is required. The technique we use is as follows:

First of all, let $\left\{x_{1}, x_{2}, \ldots, x_{N}, \ldots\right\}\left(x_{i}=\left[x_{i, 1}, x_{i, 2}, \ldots, x_{i, M}\right]^{T}\right)$ be a particular data stream in a $M$ dimensional real space, $\mathrm{s}^{M}$. The subscript $i$ denotes the time instance at which $x_{i}$ arrives. It is assumed that the data stream is composed of samples of $C$ different categories/classes, and, thus, the stream can be divided into $C$ sub-data streams in accordance to the categories that the data 
The ALMMo-0* neuro-fuzzy system, as illustrated in Figure 1, is composed of $C$ parallel IF...THEN rules, each of which corresponds to one of the $C$ categories and has the following form $(c=1,2, \ldots, C)[33$ :

$$
\begin{array}{r}
\text { IF }\left(x \sim p_{c}^{1}\right) \text { OR }\left(x \sim p_{c}^{2}\right) \text { OR } \ldots \text { OR }\left(x \sim p_{c}^{P_{c}}\right) \\
\text { THEN }(\text { category } c)
\end{array}
$$

151

samples belong to (one sub-stream per category). At the $N^{\text {th }}$ time instance, the $c^{t h}$ sub data-stream is denoted as $\left\{x_{c, 1}, x_{c, 2}, \ldots, x_{c, N_{c}}\right\}$, where $c=1,2, \ldots, C$ and $\sum_{c=1}^{C} N_{c}=N$. Unless specifically declared otherwise, all the mathematical derivations in the remainder of this paper are conducted at the $N^{\text {th }}$ time instance by default.

\subsection{Architecture}

The ALMMo-0* is build upon on the ALMMo-0 neuro-fuzzy system 33. which based on the zero-order parallel IF...THEN rules of AnYa type 43]. The general architecture of the ALMMo-0* is given in Figure1. Figure11(a) presents the architecture of the neuro-fuzzy system during the system identification stage; Figure 1.(b) gives the system architecture during the validation stage; Figure1(c) is the zoomed-in architecture of the $c^{t h}$ parallel IF...THEN rule $(c=1,2, \ldots, C)$.

where $p_{c, j}\left(j=1,2, \ldots, P_{c}\right)$ is the $j^{t h}$ prototype of the $c^{t h}$ category; $P_{c}$ is the number of the identified prototypes in total from the observed data samples of the $c^{\text {th }}$ category.

As one can see from equation (1) and Figure 1, each parallel IF...THEN rule is built upon a number of prototypes that are identified from data samples of the corresponding sub-data stream through a nonparametric, self-organizing, self-evolving, online learning process in parallel. The prototypes are connected by the local decision-maker, which decides the output of the IF...THEN rule 


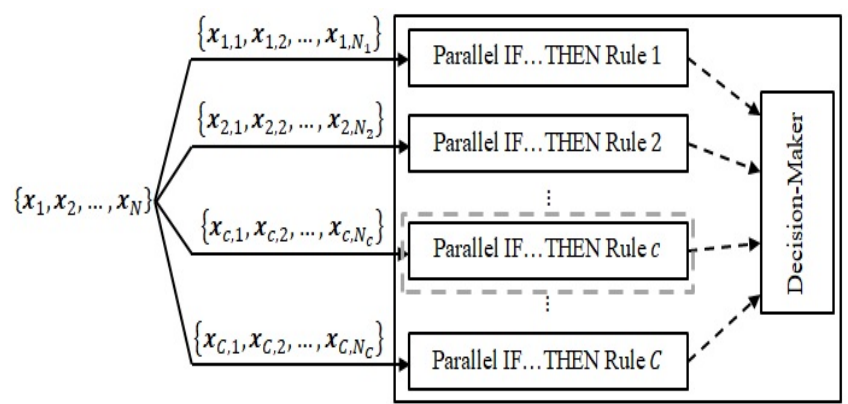

(a) The architecture for identification

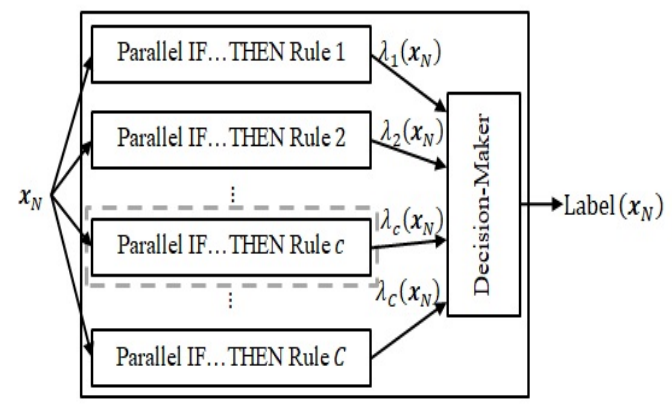

(b) The architecture for validation

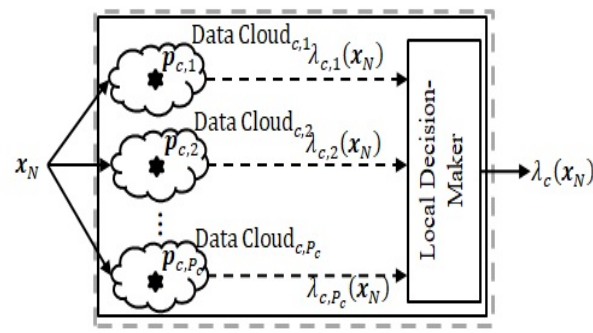

(c) Zoom-in structure of the $c^{\text {th }}$ parallel IF...THEN rule

Figure 1: The general architecture of ALMMo-0*. 
In the following two subsections, we will summarize the main steps of the system identification and validation processes. For the detailed algorithmic procedures, please refer to [33, 44.

\subsection{Identification Process}

As each parallel IF...THEN rule is identified through a independent process from others, we present the identification process of the $c^{t h}$ rule as follows $(c=1,2, \ldots, C)$. One can apply the same principle to all IF...THEN rules of the rule base.

The same principle to all IF...THEN rules of the rule base may be applied.

Step 1. Standardize the newly observed data sample, $x_{c, k}$

$$
x_{c, k}=\frac{x_{c, k}-\min _{\forall k}\left(x_{c, k}\right)}{\operatorname{std}\left(x_{c, k}\right)}
$$

Then, the data are rescaled within the range $[0,1]$ to consider variables in the same proportion. Unity-based normalization of the $c$-th element of the $k$-th sample is given by:

$$
x_{c, k}=\frac{x_{c, k}-\min _{\forall k}\left(x_{c, k}\right)}{\max _{\forall k}\left(x_{c, k}\right)-\min _{\forall k}\left(x_{c, k}\right)}
$$

If $k=1$, go to Step 2; otherwise, go to Step 3.

Step 2. Initialize the global meta-parameters with the first data sample, $x_{c, 1}$ observed:

$$
P_{c} \leftarrow 1 ; \quad \mu_{c} \leftarrow x_{c, 1} ;
$$

where $\mu_{c}$ denotes the global mean of data samples of the $c^{t h}$ category. 
Secondly, initialize the first data cloud, $\mathrm{C}_{c, 1}$ :

$$
\begin{array}{r}
\mathrm{C}_{c, 1} \leftarrow\left\{x_{c, 1}\right\} ; \quad p_{c, 1} \leftarrow x_{c, 1} ; \\
S_{c, 1} \leftarrow 1 ; \quad r_{c, 1} \leftarrow r_{o} ;
\end{array}
$$

185

Then, identify the nearest prototype $p_{c, n^{*}}$ to $x_{c, k}$ :

$$
n^{*}=\underset{j=1,2, \ldots, P_{c}}{\operatorname{argmin}}\left(\left\|x_{c, k}-p_{c, j}\right\|\right)
$$

If the following condition (equation (9) 33 is met, go to Step 4; otherwise, go to Step 5.

$$
\begin{array}{r}
I F\left(D_{c, k}\left(x_{c, k}\right)>\max _{j=1,2, \ldots, P_{c}}\left(D_{c, k}\left(p_{c, j}\right)\right)\right) \\
\text { OR }\left(D_{c, k}\left(x_{c, k}\right)<\min _{j=1,2, \ldots, P_{c}}\left(D_{c, k}\left(p_{c, j}\right)\right)\right) \\
\text { OR }\left(\left\|p_{c, n^{*}}-x_{c, k}\right\|>r_{c, n^{*}}\right) \\
\text { THEN (add a new data cloud })
\end{array}
$$

Step 3. Calculate the data density at $x_{c, k}$ and $p_{c, j}\left(j=1,2, \ldots, P_{c}\right)$ [4]:

$$
D_{c, k}(z)=\frac{1}{1+\frac{\| z-\left.\mu_{c}\right|^{2}}{1-\left\|\mu_{c}\right\|^{2}}} ;
$$

where, $z=x_{c, k}, p_{c, 1}, p_{c, 2}, \ldots, p_{c, P_{c}}$ 
Then, go to Step 6.

Step 5. Update the meta-parameters of the nearest data cloud:

$$
\begin{array}{r}
\mathrm{C}_{c, n^{*}} \leftarrow \mathrm{C}_{c, n^{*}}+\left\{x_{c, k}\right\} ; \\
p_{c, n^{*}} \leftarrow \frac{S_{c, n^{*}}}{S_{c, n^{*}}+1} p_{c, n^{*}}+\frac{S_{c, n^{*}}}{S_{c, n^{*}}+1} x_{c, k} ; \\
S_{c, n^{*}} \leftarrow S_{c, n^{*}}+1 ; \\
r_{c, n^{*}} \leftarrow \sqrt{\frac{r_{c, n^{*}}+\left(1-\left\|p_{c, n^{*}}\right\|^{2}\right)^{2}}{2}} ;
\end{array}
$$

Then, go to Step 6.

Step 6. Update the IF...THEN rule, $\mathrm{R}_{c}$ with the identified prototypes:

$$
\begin{array}{r}
\mathrm{R}_{c}: \quad \text { IF }\left(x \sim p_{c, 1}\right) \text { OR } \ldots \text { OR }\left(x \sim p_{c, P_{c}}\right) \\
\text { THEN }(\text { category } c)
\end{array}
$$

The ALMMo-0* Predict learning and estimation algorithm is summarized below.

\section{ALMMo-0*: Learning Procedure}

1: While the new data sample of the the $\mathrm{c}-t h$ class $x_{c, k}$ available

2: Standardize and Normalize $x_{c, k}$ according to equations 2 and 3

3: $\mathbf{I F ~} \mathrm{k}=1$

4: $\quad P_{c} \leftarrow 1$

5: $\quad \mu_{c} \leftarrow x_{c, 1}$;

6: $\quad C_{c, 1} \leftarrow x_{c, 1}$;

7: $\quad p_{c, 1} \leftarrow x_{c, 1}$;

8: $\quad S_{c, 1} \leftarrow 1$;

9: $\quad r_{c, 1} \leftarrow r_{o}$

10: ELSE

11: Calculate $D_{c, k}$ using equation 7

12: Update $p_{c, j}\left(j=1,2, \ldots, P_{c}\right)$ using equation 7

13: $\quad$ IF Condition (eq. 9 is satisfied THEN 


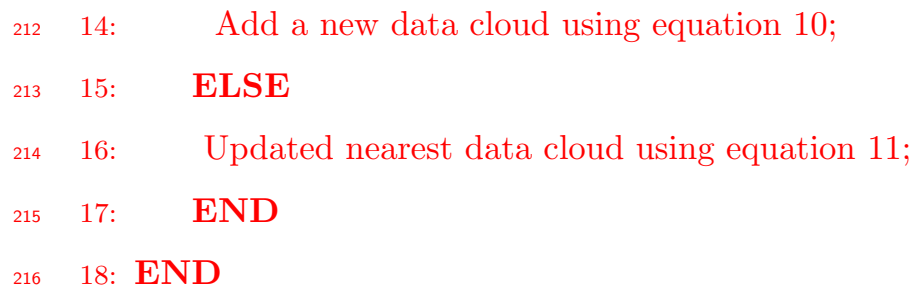

\subsection{Validation Process}

Each available validation data sample is sent to all AnYa FRB sub-classifiers corresponding to the $C$ classes of the dataset. As each class may have several AnYa type of fuzzy rules, the output, namely, the score of confidence $\lambda$ of each AnYa FRB rule is given as follows:

$$
\mathrm{R}_{c}: \quad I F\left(x \sim p_{c, 1}\right) \operatorname{THEN}\left(\lambda_{j}=\exp \left(-\frac{1}{2}\left\|x-p_{c, 1}\right\|^{2}\right)\right)
$$

The "winner takes all" operator is used to select the most confident rule and assign the validation data sample the corresponding label. In other words, each validation data sample is compared to all prototypes identified in the training phase, and a label is attached to his validation data sample according to the label of the nearest identified prototype as illustrated in Figure 2

$$
\text { Label }=\underset{j=1,2, \ldots, P}{\operatorname{argmax}}\left(\lambda_{i}\right)
$$

\section{Numerical Results}

The 'PhysioNet' dataset contains a total of 13015 samples of heart sound recordings, lasting from 5 seconds to just over 120 seconds. Recordings were collected from different locations on the body, including aortic area, pulmonic area, tricuspid area and mitral area. The collected heart sound recordings were divided into two types: normal and abnormal heart sound recordings. The normal recordings were from healthy subjects and the abnormal ones were from 


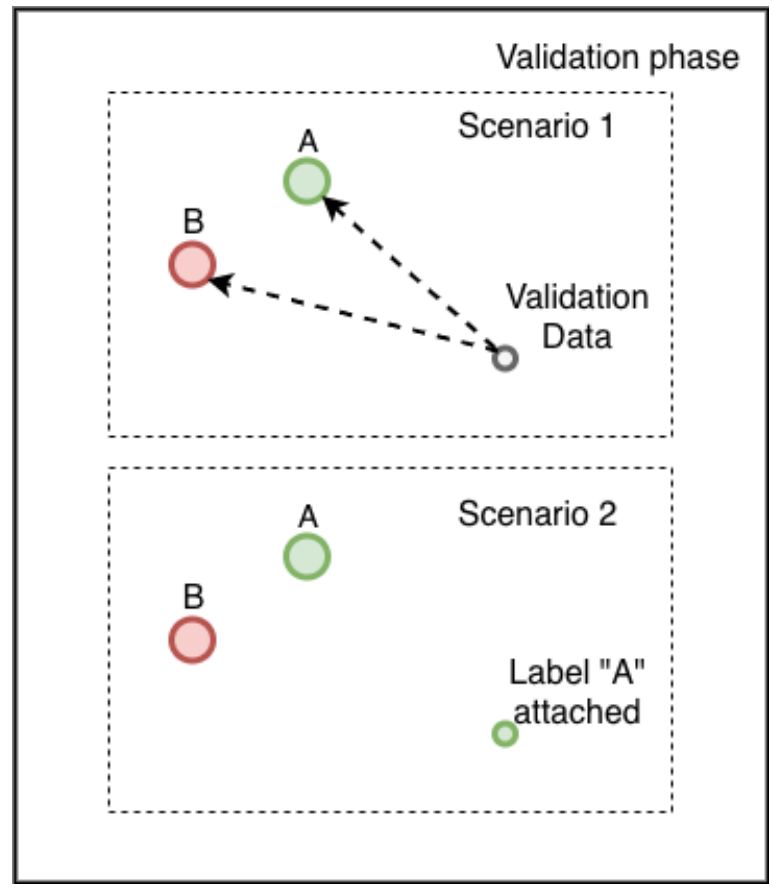

Figure 2: Validation phase illustration.

patients with a confirmed cardiac diagnosis.Figure 3 illustrates the normal and abnormal heart sound over time, while Figure 4 shows the power spectrum over the normalized frequency for both normal and abnormal heart sound conditions [39, 45].

\subsection{Pre-Processing}

The 'PhisioNet' dataset was divided into $70 \%$ for training and $30 \%$ for validation purposes. It is important to highlight that the 'PhysioNet' dataset is imbalanced as it contains 3158 samples of normal condition heart sounds and 9857 samples of abnormal sounds.

The following types of features were extracted from the heart sound recordings:

- Statistical features: mean, median, and standard deviation. 


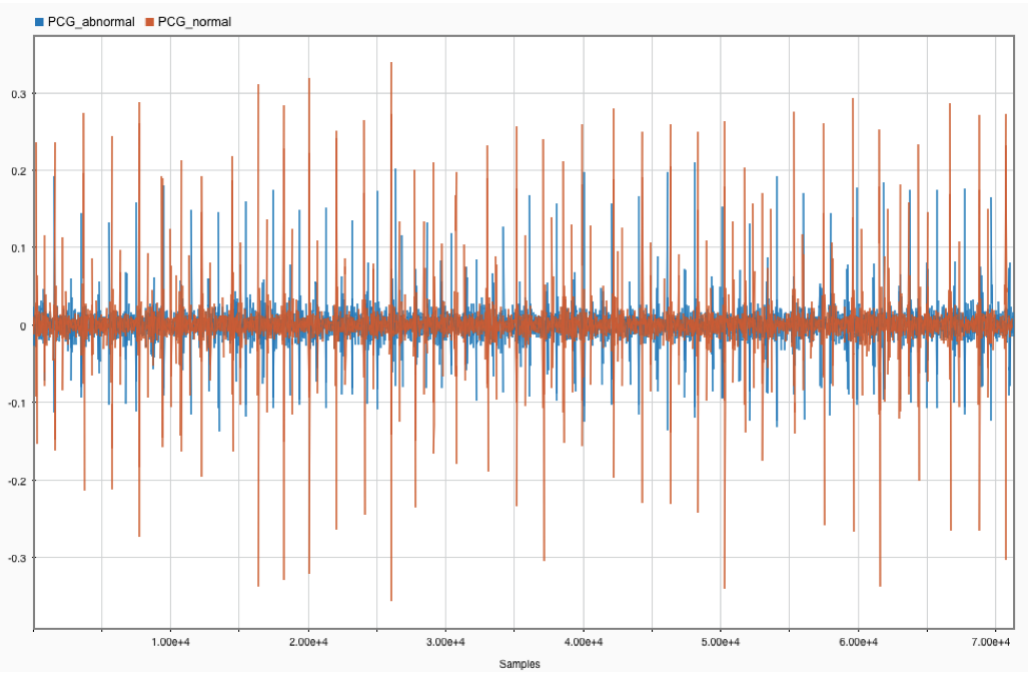

Figure 3: Normal and abnormal heart sound over time

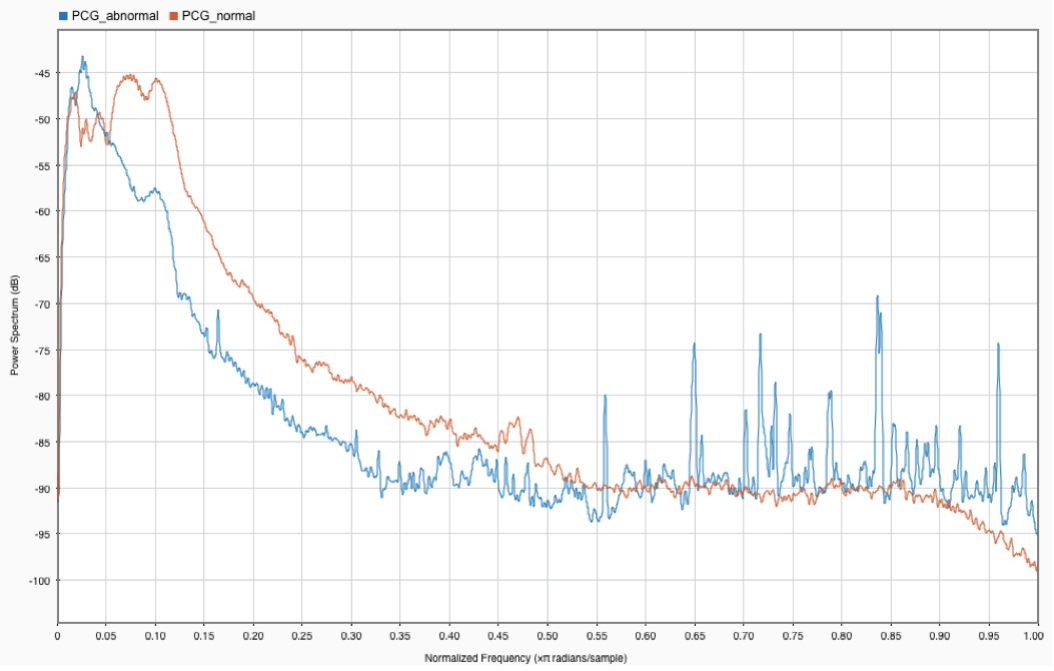

Figure 4: Power spectrum over the normalized frequency

- Signal processing features: dominant frequency, spectrum entropy, and Mel Frequency Cepstral Coefficients (MFCC).

Dominant frequency refers to the most relevant frequency in the sound spec- 
trum [46]. Spectrum entropy is defined as a measure of its spectral power distribution, and it is based on the Shannon entropy [47. Spectrum entropy treats as a probability distribution the signal's normalized power distribution in the frequency domain. Then, it calculates the Shannon entropy of it, see [48] for detailed proof for spectrum entropy.

Mel Frequency Cepstral Coefficients is a representation of the short-term power spectrum of a sound, based on a linear cosine transform of a log power spectrum on a nonlinear mel scale of frequency [49].

MFCCs are commonly derived as follows [49]:

- Divide the signals into frames

- Take the Fourier transform of each signal.

- Take the logs of the amplitude spectrum.

- Take the discrete cosine transform of the list of logs generated in the previous step.

- The MFCCs feautres are the amplitudes of the resulting spectrum.

Therefore, 27 features extracted from the audio recordings signals are described in Table 1 
Table 1: Features Summary

\begin{tabular}{c|cc}
\hline Features & Quantity & Type \\
\hline Mean & 1 & Statistical \\
Median & 1 & Statistical \\
Standard Deviation & 1 & Statistical \\
Mean Absolute Deviation & 1 & Statistical \\
Quantile 25 & 1 & Statistical \\
Quantile 75 & 1 & Statistical \\
Signal IQR & 1 & Signal Processing \\
Sample Skewness & 1 & Statistical \\
Sample Kurtosis & 1 & Statistical \\
Signal Entropy & 1 & Signal Processing \\
Spectral Entropy & 1 & Signal Processing \\
Dominant Frequency Value & 1 & Signal Processing \\
Dominant Frequency Magnitude & 1 & Signal Processing \\
Dominant Frequency Ratio & 1 & Signal Processing \\
MFCC & 13 & Signal Processing \\
\hline
\end{tabular}

\subsection{Performance Evaluation}

In order to evaluate the performance of the considered methods the following indexes are considered: sensitivity (Se), specificity (Sp), and overall score (MAcc). These indexes are calculated as:

$$
\begin{gathered}
S e=\frac{T P}{T P+F N}, \\
S p=\frac{T N}{T N+F P}, \\
M A c c=\frac{S e+S p}{2} .
\end{gathered}
$$


where $T P, F P, T N, F N$ denote true and false, negative and positive respectively.

Sensitivity is considered as an indicator of the classifier's ability to discover the true class. Specificity is considered as a index of the classifier's ability to define other classes. The overall score (MAcc) is given by the mean of sensitivity and specificity indexes.

The receiver operating characteristic (ROC) method is also considered in the analysis. As the ROC method is insensitive to both changes in class distribution and proportion of samples per class it provides a convenient way to evaluate the quality of evolving classifiers in nonstationary environment [50].

$$
\begin{aligned}
& T P_{\text {ratio }}=\frac{T P}{T P+F N} \\
& F P_{\text {ratio }}=\frac{F P}{F P+T N}
\end{aligned}
$$

Each cut-off threshold in the ROC approach corresponds to a point (sensitivity/specificity pair) in the ROC space [50. The closer the ROC curve is to the upper left corner, the better is the classification rate.

All the experiments were conducted with MATLAB 2018a using a personal computer with a $1.8 \mathrm{GHz}$ Intel Core i5 processor, 8-GB RAM, and MacOS operating system. The classification experiments were executed using 10-fold cross validation under the same ratio of training-to-testing sample sets. The proposed approach is compared with results obtained by Computing in Cardiology Challenge winners in order to determine the efficiency of the proposed model. Data and methods used in this research are available upon request.

\subsection{Classification Results}

In this section we will demonstrate the results obtained for heart sounds classification. Computational simulations were performed to assess the accuracy of the classification methods considering heart sounds recordings. Table ?? summarizes the results obtained by the proposed ALMMo- $0^{*}$ and its competitors 
considering the 'Classification of Normal/Abnormal Heart Sound Recordings' dataset provided by Phisionet. Were considered 27 features inputs in the data space in order to determine if the patient heart sound is classified as normal or abnormal. Initial parameters were set in order that the final structure of the ALMMo- $0^{*}$, and ALMMo-0 contained a reasonable amount of identified prototypes, improving interpretability of the final model. The following parameter was chosen: $r_{o}=\sqrt{2-2 \cos \left(30^{\circ}\right)}$ for the ALMMo- $0^{*}$, and ALMMo-0 neurofuzzy classifiers.

Table 2: Performance Comparasion: Heart sound classification

\begin{tabular}{c|ccc}
\hline Method & Sensitivity $($ Se $)$ & Specificity $($ Sp $)$ & MAcc \\
\hline ALMMo-0* & 0.9082 & $\underline{\mathbf{0 . 9 5 2 6}}$ & $\underline{\mathbf{0 . 9 3 0 4}}$ \\
ALMMo-0 & 0.7930 & 0.9430 & 0.8680 \\
AdaBoost \& CNN [6] & $\underline{\mathbf{0 . 9 4 2 4}}$ & 0.7781 & 0.8602 \\
Ensemble of SVMs [6] & 0.8691 & 0.8490 & 0.8590 \\
Regularized Neural Network [6] & 0.8743 & 0.8297 & 0.8520 \\
MFCCs, Wavelets, Tensors \& KNN [6] & 0.8639 & 0.8269 & 0.8454 \\
Random Forest + LogitBoost [6] & 0.8848 & 0.8048 & 0.8448 \\
Ensemble of neural networks [51] & 0.8982 & 0.9253 & 0.9117 \\
Deep Structured Features [10] & 0.8450 & 0.8690 & 8380 \\
Matrix norm sparse coding + 20 time-domain features [52] & 0.8867 & 0.8816 & 0.8841 \\
\hline
\end{tabular}

Table 2 shows that the ALMMo-0* approach has the higher accuracy performance. ALMMo- $0^{*}$ could obtain better results in terms of $S p$ and $M A c c$ than its competitors, including ALMMo-0. The AdaBoost \& CNN could obtain a better performance in terms of sensitity, in other words, it had a better ability to discover the true class. However, ALMMo- $0 *$ showed a better performance in terms of specificity (classifier's ability to define other classes), due to its prototype-based nature. Moreover, it had the second best result in terms of sensitivity. Therefore, the proposed approach could obtain the best result in 

mance of the best considered approaches.

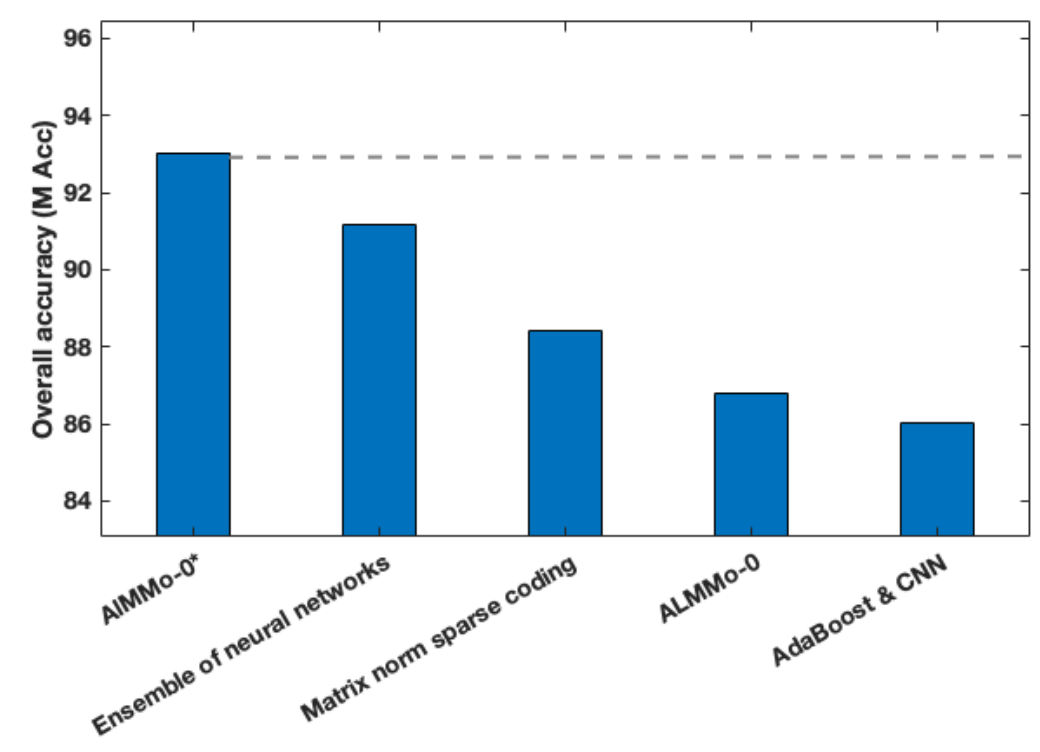

Figure 5: Overall accuracy performance of the best considered approaches

terms of overall score $(M A c c)$. Figure 5 illustrates the overall accuracy perfor-

The area under the ROC curves confirms that ALMMo-0 is able to work efficiently in this classification problem, no matter if the distribution is changed to any other distribution or if the dataset is imbalanced. The area above the ALMMo-0 ROC curve refers in part to $5.81 \%$ of classification error with different assigned labels.

The prototypes identified by ALMMo-0* are visualized in Figure 7 , where the first two principal components are used for visual clarity. Voronoi tessellations are created by using these prototypes to attract nearby data samples forming data clouds. Thanks to its prototype-based nature, medical doctors can easily identify abnormal heart sounds by comparing a patient's sample with the identified prototypes from abnormal samples by ALMMo-0* (also see Figure 7). 


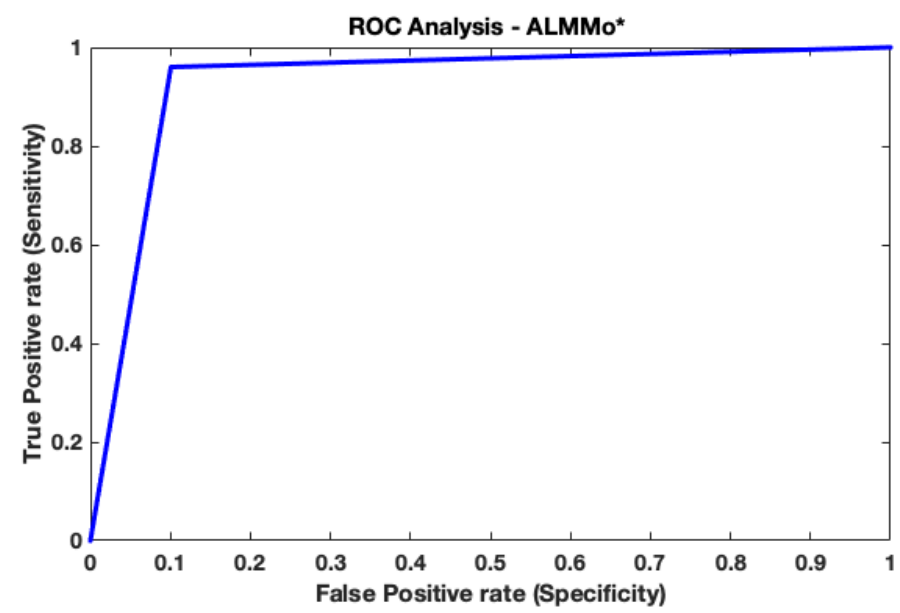

Figure 6: ROC analysis for heart sound classification using ALMMo-0*

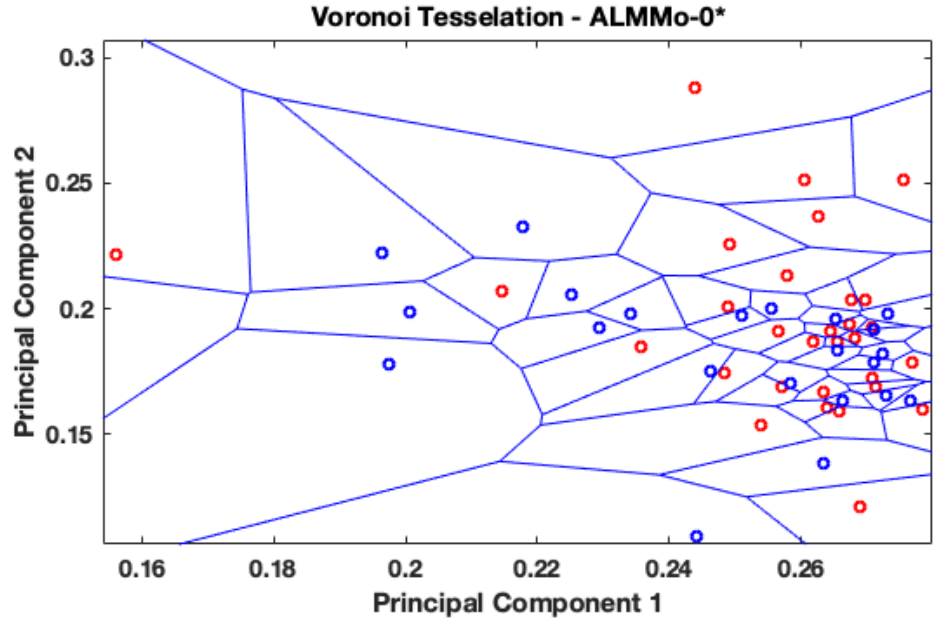

Figure 7: Voronoi Tesselation of the identified prototypes - ALMMo-0*

AnYa type fuzzy rules generated by the ALMMo-0* model provide a very intuitive representation for specialists. Moreover, each of the AnYa type fuzzy rules can be interpreted as a number of simpler fuzzy rules with single prototype connected by 'OR' operators. As a result, a massive parallelization is possible. The transparent process provided by the ALMMo-0* model supports under- 
standability of the system, differing from other machine learning approaches, which are called 'black box', since they hide (due to its nature) from users all the insights used to generate the final resulting structure.

AnYa fuzzy rule for the Normal class in ALMMo-0* top layer can be written as following:

$$
\begin{gathered}
\text { IF }\left(\mathrm{x} \sim\left\{p_{1}^{1}\right\}\right) \text { OR }\left(x \sim\left\{p_{1}^{2}\right\}\right) \text { OR }\left(x \sim\left\{p_{1}^{3}\right\}\right) \text { OR } \ldots \text { OR }\left(x \sim\left\{p_{1}^{20}\right\}\right) \\
\text { THEN 'Normal heart sound' }
\end{gathered}
$$

4

The prototypes identified for the 'Normal heart sound' rule are demonstrated on Table 3 .

In short, experiments have shown that the proposed deep neuro-fuzzy modeling is an efficient framework for heart sound classification tasks. Classification accuracies were higher than those produced by state-of-the-art approaches considered for this problem. The proposed ALMMo-0* could also achieve better results than achieve better results than ALMMo-0. Differently from the stateof-the-art approaches which are 'black box', the proposed method produced transparent linguistic fuzzy rules, which are human interpretable, and helpful for specialists to make a full diagnosis about the patient situation.

Generally, time to process data and adapt a fuzzy model is not a constraint for the classification problems. However, it may be an issue in higher-frequency data streams applications in real-time, as heart sound classification. ALMMo0* adaptation deals with nonstationarities very efficiently and fast. Therefore, ALMMo-0 becomes interesting for real-time sound classification scenarios.

\section{Conclusion}

In this paper, we propose an extended version of the zero-order Autonomous Learning Multiple-Model neuro-fuzzy classifier in order to classify heart sounds recordings. The proposed method extends the recently introduced ALMMo-0 classifier by adding a standardization and normalization pre-processing structure, which improves the accuracy of the method as illustrated in the analysis. 
The proposed method could obtain better results in terms of classification accuracy than the state-of-the-art methods for this type of problem. Moreover, the proposed autonomous learning neuro-fuzzy classifier has demonstrated to be able to self-adapt its structure and provide human-understandable $I F \ldots$ THEN fuzzy rule-based system structure. Rules generated may support specialists in order to make a deeper diagnosis of the patient situation. Due to its prototypebased the proposed method showed a better performance in terms of specificity (classifier's ability to define other classes), and also a better overall score result. ALMMo- $0 *$ is able to deal with the data without making any prior assumptions or training any parameters, differently from its competitors as the Convolutional Neural Networwk approach.

Future research will concentrate on the development of hierarchical structures, in order to favor the human interpretability of the results obtained. Furthermore, a density-based feature will be proposed to select the best features that explains the problem, and also provide more interpretable results for specialists.

\section{References}

[1] R. O. Duda, P. E. Hart, D. G. Stork, Pattern classification, John Wiley \& Sons, 2012.

[2] F. Safara, S. Doraisamy, A. Azman, A. Jantan, A. R. A. Ramaiah, Multilevel basis selection of wavelet packet decomposition tree for heart sound classification, Computers in biology and medicine 43 (10) (2013) 1407-1414.

[3] S. Babaei, A. Geranmayeh, Heart sound reproduction based on neural network classification of cardiac valve disorders using wavelet transforms of pcg signals, Computers in biology and medicine 39 (1) (2009) 8-15.

[4] Z. Dokur, T. Ölmez, Heart sound classification using wavelet transform and incremental self-organizing map, Digital Signal Processing 18 (6) (2008) 951-959. 
[5] W. H. Organization, et al., Noncommunicable diseases: progress monitor 2017.

[6] G. D. Clifford, C. Liu, B. Moody, D. Springer, I. Silva, Q. Li, R. G. Mark, Classification of normal/abnormal heart sound recordings: The physionet/computing in cardiology challenge 2016, in: Computing in Cardiology Conference (CinC), 2016, IEEE, 2016, pp. 609-612.

[7] T. R. Reed, N. E. Reed, P. Fritzson, Heart sound analysis for symptom detection and computer-aided diagnosis, Simulation Modelling Practice and Theory 12 (2) (2004) 129-146.

[8] F. M. Noman, S.-H. Salleh, C.-M. Ting, S. B. Samdin, H. Ombao, H. Hussain, A markov-switching model approach to heart sound segmentation and classification, IEEE journal of biomedical and health informatics.

[9] M. J. Sarnak, A. S. Levey, A. C. Schoolwerth, J. Coresh, B. Culleton, L. L. Hamm, P. A. McCullough, B. L. Kasiske, E. Kelepouris, M. J. Klag, et al., Kidney disease as a risk factor for development of cardiovascular disease: a statement from the american heart association councils on kidney in cardiovascular disease, high blood pressure research, clinical cardiology, and epidemiology and prevention, Circulation 108 (17) (2003) 2154-2169.

[10] M. Tschannen, T. Kramer, G. Marti, M. Heinzmann, T. Wiatowski, Heart sound classification using deep structured features, in: Computing in Cardiology Conference (CinC), 2016, IEEE, 2016, pp. 565-568.

[11] I. Maglogiannis, E. Loukis, E. Zafiropoulos, A. Stasis, Support vectors machine-based identification of heart valve diseases using heart sounds, Computer methods and programs in biomedicine 95 (1) (2009) 47-61.

[12] Y. Koçyiğit, Heart sound signal classification using fast independent component analysis, Turkish Journal of Electrical Engineering \& Computer Sciences 24 (4) (2016) 2949-2960. 
[13] C. Rudin, Stop explaining black box machine learning models for high stakes decisions and use interpretable models instead, Nature Machine Intelligence 1 (5) (2019) 206.

[14] E. Soares, P. Angelov, B. Costa, M. Castro, Actively semi-supervised deep rule-based classifier applied to adverse driving scenarios, in: 2019 International Joint Conference on Neural Networks (IJCNN), 2019, pp. 1-8. doi:10.1109/IJCNN.2019.8851842

[15] P. Angelov, E. Soares, Towards explainable deep neural networks (xdnn) (2019). arXiv: 1912.02523.

[16] C. Potes, S. Parvaneh, A. Rahman, B. Conroy, Ensemble of feature-based and deep learning-based classifiers for detection of abnormal heart sounds, in: 2016 Computing in Cardiology Conference (CinC), IEEE, 2016, pp. $621-624$.

[17] T.-E. Chen, S.-I. Yang, L.-T. Ho, K.-H. Tsai, Y.-H. Chen, Y.-F. Chang, Y.H. Lai, S.-S. Wang, Y. Tsao, C.-C. Wu, S1 and s2 heart sound recognition using deep neural networks, IEEE Transactions on Biomedical Engineering 64 (2) (2016) 372-380.

[18] W. Zhang, J. Han, S. Deng, Heart sound classification based on scaled spectrogram and tensor decomposition, Expert Systems with Applications 84 (2017) 220-231.

[19] D. Leite, P. Costa, F. Gomide, Evolving granular neural network for semisupervised data stream classification, in: Neural Networks (IJCNN), The 2010 International Joint Conference on, IEEE, 2010, pp. 1-8.

[20] S. Silva, P. Costa, M. Santana, D. Leite, Evolving neuro-fuzzy network for real-time high impedance fault detection and classification, Neural Computing and Applications 1-14.

[21] P. P. Angelov, X. Zhou, Evolving fuzzy-rule-based classifiers from data streams, IEEE Transactions on Fuzzy Systems 16 (6) (2008) 1462-1475. 
[22] E. Soares, P. Angelov, Novelty detection and learning from extremely weak supervision, arXiv preprint arXiv:1911.00616.

[23] E. Soares, P. Costa Jr, B. Costa, D. Leite, Ensemble of evolving data clouds and fuzzy models for weather time series prediction, Applied Soft Computing 64 (2018) 445-453.

[24] D. Leite, R. Ballini, P. Costa, F. Gomide, Evolving fuzzy granular modeling from nonstationary fuzzy data streams, Evolving Systems 3 (2) (2012) 6579.

[25] J. de Jesús Rubio, Evolving intelligent algorithms for the modelling of brain and eye signals, Applied Soft Computing 14 (2014) 259-268.

[26] B. S. J. Costa, P. P. Angelov, L. A. Guedes, Real-time fault detection using recursive density estimation, Journal of Control, Automation and Electrical Systems 25 (4) (2014) 428-437.

[27] A. Lemos, D. Leite, L. Maciel, R. Ballini, W. Caminhas, F. Gomide, Evolving fuzzy linear regression tree approach for forecasting sales volume of petroleum products, in: Fuzzy Systems (FUZZ-IEEE), 2012 IEEE International Conference on, IEEE, 2012, pp. 1-8.

[28] I. Škrjanc, J. Iglesias, A. Sanchis, D. Leite, E. Lughofer, F. Gomide, Evolving fuzzy and neuro-fuzzy approaches in clustering, regression, identification, and classification: A survey, Information Sciences (2019) 344-368.

[29] D. Leite, P. Costa, F. Gomide, Evolving granular neural networks from fuzzy data streams, Neural Networks 38 (2013) 1-16.

[30] N. K. Kasabov, Neucube: A spiking neural network architecture for mapping, learning and understanding of spatio-temporal brain data, Neural Networks 52 (2014) 62-76.

[31] S. Lekkas, L. Mikhailov, Evolving fuzzy medical diagnosis of pima indians diabetes and of dermatological diseases, Artificial Intelligence in Medicine 50 (2) (2010) 117-126. 
[32] M. E. Futschik, A. Reeve, N. Kasabov, Evolving connectionist systems for knowledge discovery from gene expression data of cancer tissue, Artificial Intelligence in Medicine 28 (2) (2003) 165-189.

[33] P. Angelov, X. Gu, Autonomous learning multi-model classifier of 0-order (ALMMo-0), in: 2017 Evolving and Adaptive Intelligent Systems (EAIS), IEEE, 2017, pp. 1-7.

[34] P. P. Angelov, X. Gu, J. C. Príncipe, Autonomous learning multimodel systems from data streams, IEEE Transactions on Fuzzy Systems 26 (4) (2018) 2213-2224.

[35] S. R. Soloman, S. S. Sawilowsky, Impact of rank-based normalizing transformations on the accuracy of test scores, Journal of Modern Applied Statistical Methods 8 (2) (2009) 9.

[36] P. Angelov, R. Yager, Simplified fuzzy rule-based systems using nonparametric antecedents and relative data density, in: 2011 IEEE Workshop on Evolving and Adaptive Intelligent Systems (EAIS), IEEE, 2011, pp. $62-69$.

[37] P. P. Angelov, X. Gu, Deep rule-based classifier with human-level performance and characteristics, Information Sciences 463-464 (2018) 196-213.

[38] X. Gu, P. P. Angelov, Semi-supervised deep rule-based approach for image classification, Applied Soft Computing 68 (2018) 53-68.

[39] C. Liu, D. Springer, Q. Li, B. Moody, R. A. Juan, F. J. Chorro, F. Castells, J. M. Roig, I. Silva, A. E. Johnson, et al., An open access database for the evaluation of heart sound algorithms, Physiological Measurement 37 (12) (2016) 2181.

[40] S. Kotsiantis, D. Kanellopoulos, P. Pintelas, Data preprocessing for supervised leaning, International Journal of Computer Science 1 (2) (2006) 111-117. 
[41] J. C. Russ, The image processing handbook, CRC press, 2016.

[42] P. Angelov, D. P. Filev, N. Kasabov, Evolving intelligent systems: methodology and applications, John Wiley \& Sons, 2010.

[43] P. Angelov, R. Yager, A new type of simplified fuzzy rule-based system, International Journal of General Systems 41 (2) (2012) 163-185.

[44] P. P. Angelov, X. Gu, Empirical approach to machine learning, Springer, 2018.

[45] P. PhysioToolkit, Physionet: components of a new research resource for complex physiologic signals, Circulation. v101 i23. e215-e220.

[46] F. Atienza, J. Almendral, J. Jalife, S. Zlochiver, R. Ploutz-Snyder, E. G. Torrecilla, A. Arenal, J. Kalifa, F. Fernández-Avilés, O. Berenfeld, Realtime dominant frequency mapping and ablation of dominant frequency sites in atrial fibrillation with left-to-right frequency gradients predicts long-term maintenance of sinus rhythm, Heart Rhythm 6 (1) (2009) 33-40.

[47] V. Sharma, A. Parey, A review of gear fault diagnosis using various condition indicators, Procedia Engineering 144 (2016) 253-263.

[48] Y. Pan, J. Chen, X. Li, Spectral entropy: a complementary index for rolling element bearing performance degradation assessment, Proceedings of the Institution of Mechanical Engineers, Part C: Journal of Mechanical Engineering Science 223 (5) (2009) 1223-1231.

[49] B. Logan, et al., Mel frequency cepstral coefficients for music modeling., in: International Society for Music Information Retrieval (ISMIR), Vol. 270, 2000, pp. 1-11.

[50] T. Fawcett, An introduction to roc analysis, Pattern recognition letters 27 (8) (2006) 861-874. 
[51] M. Zabihi, A. B. Rad, S. Kiranyaz, M. Gabbouj, A. K. Katsaggelos, Heart sound anomaly and quality detection using ensemble of neural networks without segmentation, in: 2016 Computing in Cardiology Conference (CinC), IEEE, 2016, pp. 613-616.

[52] B. M. Whitaker, P. B. Suresha, C. Liu, G. D. Clifford, D. V. Anderson, Combining sparse coding and time-domain features for heart sound classification, Physiological measurement 38 (8) (2017) 1701. 
Table 3: Identified Prototypes for the 'Normal heart sound' rule

\begin{tabular}{|c|c|c|c|c|}
\hline Features & $p_{1}^{1}$ & $p_{1}^{2}$ & $p_{1}^{3}$ & $p_{1}^{20}$ \\
\hline$f_{1}$ & $-2.7121 \mathrm{e}-05$ & $1.5511 \mathrm{e}-04$ & $-8.0804 \mathrm{e}-05$ & $-5.4135 \mathrm{e}-05$ \\
\hline$f_{2}$ & $1.5259 \mathrm{e}-04$ & 0.0013 & 0 & $-1.2207 \mathrm{e}-04$ \\
\hline$f_{3}$ & 0.0203 & 0.0795 & 0.0167 & 0.0096 \\
\hline$f_{4}$ & 0.0123 & 0.0441 & 0.0099 & 0.0057 \\
\hline$f_{5}$ & -0.0084 & -0.0220 & -0.0067 & -0.0034 \\
\hline$f_{6}$ & 0.0082 & 0.0237 & 0.0063 & 0.0030 \\
\hline$f_{7}$ & 0.0166 & 0.0457 & 0.0130 & 0.0064 \\
\hline$f_{8}$ & 1.4484 & -0.4713 & 0.0276 & 0.2136 \\
\hline$f_{9}$ & 21.1471 & 15.7475 & 22.7916 & 15.4637 \\
\hline$f_{10}$ & -2.7659 & -1.5085 & -2.9793 & -3.5261 \\
\hline$f_{11}$ & 0.2868 & 0.3124 & 0.4749 & 0.3184 \\
\hline$f_{12}$ & 17.0982 & 41.0357 & 35.6619 & 21.0064 \\
\hline$f_{13}$ & 0.0669 & 0.0439 & 0.0278 & 0.0633 \\
\hline$f_{14}$ & 0.2244 & 0.2951 & 0.1133 & 0.2680 \\
\hline$f_{15}$ & 88.1961 & 100.0686 & 92.9163 & 87.5767 \\
\hline$f_{16}$ & 7.3405 & 2.4487 & 4.5780 & 5.2651 \\
\hline$f_{17}$ & 6.4674 & 7.0189 & -2.6415 & -4.2657 \\
\hline$f_{18}$ & -0.0512 & 1.3058 & -1.1482 & 6.2212 \\
\hline$f_{19}$ & -2.5149 & -2.9223 & -3.8693 & 4.6041 \\
\hline$f_{20}$ & -3.1430 & -2.3074 & -6.2024 & -4.0199 \\
\hline$f_{21}$ & -1.9638 & 0.8658 & -6.2406 & -7.7832 \\
\hline$f_{22}$ & -0.1132 & -4.5618 & -3.1221 & -3.3297 \\
\hline$f_{23}$ & -0.2849 & -5.7582 & 0.8459 & -0.3391 \\
\hline$f_{24}$ & 1.6218 & 0.9306 & -0.6360 & -0.1036 \\
\hline$f_{25}$ & -0.5334 & -3.0779 & -0.6840 & -2.0954 \\
\hline$f_{26}$ & -1.6926 & -2.3390 & 1.9931 & -3.0208 \\
\hline$f_{27}$ & -2.0239 & -0.8391 & 0.6190 & -0.9700 \\
\hline
\end{tabular}

